\title{
Electron-phonon interaction in freely suspended quantum dots
}

\author{
Eva M. Höhberger ${ }^{\mathrm{a}, *}$, Jochen Kirschbaumª ${ }^{\mathrm{a}}$ Robert H. Blick ${ }^{\mathrm{a}}$, Jörg P. Kotthaus ${ }^{\mathrm{a}}$, \\ Werner Wegscheider ${ }^{b}$ \\ ${ }^{a}$ Center for NanoScience and Sektion Physik, Ludwig-Maximilians-Universität, 80539 München, Germany \\ ${ }^{\mathrm{b}}$ Institut für Angewandte and Experimentelle Physik, Universität Regensburg, 93040 Regensburg, Germany
}

\begin{abstract}
We present work on single and double quantum dots which have been defined in the two-dimensional electron gas of a freely suspended GaAs/AlGaAs heterostructure. The dots are formed either by pairs of etched constrictions or by gating electrodes and characterized in Coulomb blockade measurements. Because of their small overall dimensions the suspended dots constitute phonon cavities in which electron transport is strongly modified by electron-phonon interaction. Our measurements indicate that the emission of phonons suppresses linear transport in the Coulomb blockade regime. Upon application of a perpendicular magnetic field Coulomb blockade oscillations reemerge, as well as under nonlinear transport conditions.
\end{abstract}

PACS: 73.23.Hk; 85.85.+j; 71.38.-k; 62.25.+g

Keywords: Quantum dots; Electron-phonon interaction; Freely suspended nanostructures; NEMS

Achieving long dephasing times is of fundamental importance for studying quantum mechanical properties of mesoscopic systems. Being only weakly coupled to the environment, quantum dots are well-suited tools for accomplishing this task [1,2]. However, gaining control of the decoherence mechanisms in these systems requires a detailed understanding of the dissipation processes such as electron-phonon scattering which has been shown to strongly modify the transport properties of quantum dots even at low temperatures $[3,4]$. The ongoing progress of three-dimensional nanostructuring enables an even more profound analysis of these processes since in freely suspended quantum dots not only the electronic states but also the phonon modes are confined [5]. Recently, Debald et al. pointed out that in such a phonon cavity the phonon emission and hence the degree of dephasing can be actively adjusted varying the inter-dot coupling of a double dot [6].

Here we report on the fabrication of tunable single and double quantum dots which have been realized in a freely suspended semiconductor membrane. Coulomb blockade

\footnotetext{
* Corresponding author.

E-mail address: eva.hoehberger@physik.uni-muenchen.de (E.M. Höhberger).
}

observed in the single dots is discussed and possible effects of phonon confinement are pointed out.

The quantum dots are defined in the two-dimensional electron gas (2DEG) of a GaAs/AlGaAs heterostructure located $40 \mathrm{~nm}$ below the sample surface. The 2DEG is embedded in a $130 \mathrm{~nm}$ thick membrane followed by the sacrificial layer consisting of $400 \mathrm{~nm}$ of $\mathrm{Al}_{0.8} \mathrm{Ga}_{0.2}$ As separating the active layer from the buffer. In order to obtain freely suspended nanostructures a series of process steps involving two subsequent steps of electron beam lithography and a combination of dry and wet etching techniques is performed which has been described in more detail previously [7-9].

Two complementing approaches have been utilized to generate freely suspended quantum dots: In Fig. 1(a) symmetric indentations in the 2DEG form a cavity, the electronic properties of which can be modified by a nearby gate electrode. Fig. 1(b) displays a double dot defined in a straight suspended beam with the aid of three Schottky gates crossing the structure. In both cases, the underlying beam has a lithographic width of $600 \mathrm{~nm}$ which is reduced by edge depletion of the 2DEG resulting in an electronic width of $450 \mathrm{~nm}$.

In Fig. 2(a) the differential conductance of the single dot from Fig. 1(a) with respect to both the gate voltage $V_{\mathrm{g}}$ and 


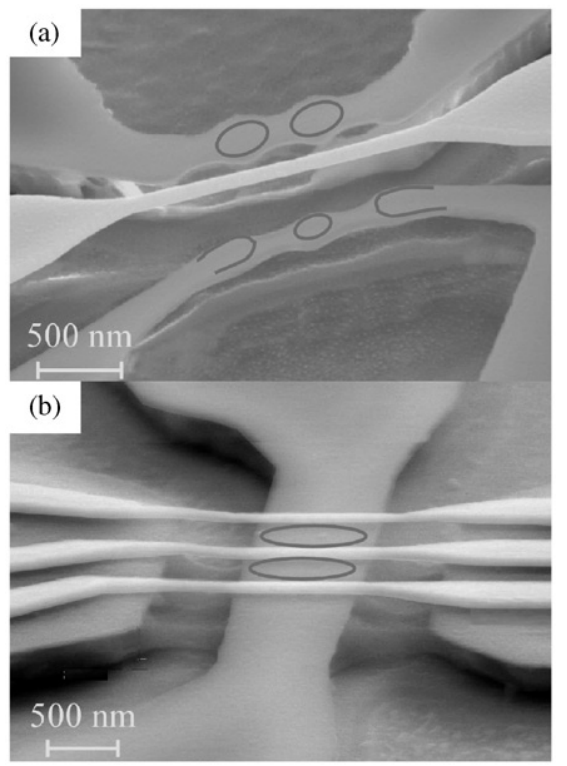

Fig. 1. Freely suspended quantum dot structures defined by (a) symmetric indentations and (b) crossing gate electrodes.

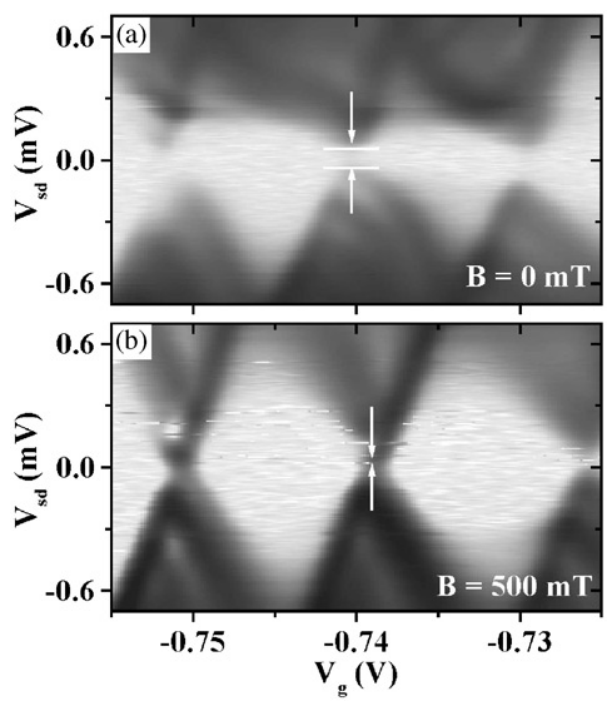

Fig. 2. (a) Coulomb blockade in a freely suspended quantum dot. The observed energy gap is marked by arrows. (b) At a magnetic field of $B=500 \mathrm{mT}$ linear transport is re-established.

source drain voltage $V_{\text {sd }}$ is shown in a gray scale plot (white: $0.02 \mu \mathrm{S}$; black: $12 \mu \mathrm{S}$ ). Measurements were performed in a dilution refrigerator at a bath temperature of $10 \mathrm{mK}$ cooling the electron system to $100 \mathrm{mK}$. Clear Coulomb blockade diamonds are resolved, corresponding to a charging energy of $E_{\mathrm{C}}=0.56 \mathrm{meV}$ and a dot capacitance of $C_{\Sigma}=140 \mathrm{aF}$.
Approximating the dot as a circular disk with a capacitance of $C_{\Sigma}=8 \varepsilon_{r} \varepsilon_{0} R$ using $\varepsilon_{\mathrm{r}}=13$ for GaAs the effective radius of the depleted dot can be determined to be $R \approx 160 \mathrm{~nm}$.

However, a striking contradiction to Coulomb blockade theory is found in linear transport as single electron tunneling for vanishing source drain voltages is not observed. As marked exemplarily by the arrows at $V_{\mathrm{g}}=-0.74 \mathrm{~V}$ in Fig. 2(a) an energy gap of $E_{\mathrm{g}}=100 \mu \mathrm{eV}$ is opened. We attribute this behavior to inelastic electron-phonon scattering in the freely suspended membrane misaligning the discrete electron levels from the reservoirs, noting that the lowest energy for an acoustic phonon confined in a membrane of $130 \mathrm{~nm}$ thickness is comparable to $E_{\mathrm{g}}$. In Fig. 2(b) the same measurement was performed for a perpendicular magnetic field of $B=500 \mathrm{mT}$. Here, the borders of the Coulomb diamonds show distinct crossings at $V_{\mathrm{sd}}=0 \mathrm{mV}$ corresponding to a re-established single electron tunneling behavior which might be explained by a quasi-continuum of excited states replacing the missing ground state.

In conclusion, we have realized quantum dots in a freely suspended membrane and demonstrated Coulomb blockade. An additional energy gap blocking linear transport might be related to the not only electronically but also phononically confined geometry.

Funds from the Bundesministerium für Forschung und Technologie (BMBF) within contract 01BM914 are gratefully acknowledged.

\section{References}

[1] A. Yacoby, M. Heiblum, D. Mahalu, H. Shtrikman, Phys. Rev. Lett. 74 (1995) 4047;

E. Buks, R. Schuster, M. Heiblum, D. Mahalu, V. Umansky, Nature 391 (1998) 871.

[2] A.W. Holleitner, C.R. Decker, H. Qin, K. Eberl, R.H. Blick, Phys. Rev. Lett. 87 (2001) 256802;

A.W. Holleitner, R.H. Blick, A.K. Hüttel, K. Eberl, J.P. Kotthaus, Science 297 (2002) 70.

[3] T. Fujisawa, T.H. Oosterkamp, W.G. van der Wiel, B.W. Broer, R. Aguado, S. Tarucha, L.P. Kouwenhoven, Science 282 (1998) 932;

H. Qin, A.W. Holleitner, K. Eberl, R.H. Blick, Phys. Rev. B 64 (2001) 241302.

[4] T. Brandes, B. Kramer, Phys. Rev. Lett. 83 (1999) 3021.

[5] S. Debald, T. Vorrath, T. Brandes, B. Kramer, Proceedings of the International Conference on Physics Semiconductors, Osaka, 2000, Springer Proceedings in Physics, Vol. 87, Springer, Berlin, 2000, p. 1049.

[6] S. Debald, T. Brandes, B. Kramer, Phys. Rev. B 66 (2002) 041301(R).

[7] R.H. Blick, F.G. Monzon, W. Wegscheider, M. Bichler, F. Stern, M.L. Roukes, Phys. Rev. B 62 (2000) 17103.

[8] E.M. Höhberger, R.H. Blick, F.W. Beil, W. Wegscheider, M. Bichler, J.P. Kotthaus, Physica E 12 (2002) 487.

[9] J. Kirschbaum, E.M. Höhberger, R.H. Blick, W. Wegscheider, M. Bichler, Appl. Phys. Lett. 81 (2002) 280. 